\title{
Current status and development trend of miRNAs in osteoporosis-related research: A bibliometric analysis
}

\author{
Li Yun ${ }^{1,2 \#, ~ L i n i n g ~ W a n g ~}{ }^{1,2,3 \#}$, Yalan Pan ${ }^{1,4}$, Mengmin Liu, \\ Guanglu Yang ${ }^{1,5}$, Jirimutu Xiao ${ }^{1}$, Yang Guo ${ }^{1,5}$, Yong Ma ${ }^{1,2,5}$
}

'Laboratory of New Techniques of Restoration and Reconstruction, Institute of Traumatology and Orthopedics, Nanjing University of Chinese Medicine, Nanjing, China

${ }^{2}$ School of Chinese Medicine, School of Integrated Chinese and Western Medicine, Nanjing University of Chinese Medicine, Nanjing, China

${ }^{3}$ Chinese Medicine Centre (International Collaboration between Western Sydney University and Beijing University of Chinese Medicine), Western Sydney University, Sydney, Australia ${ }^{4} \mathrm{TCM}$ Nursing Intervention Laboratory of Chronic Disease Key Laboratory, Nanjing University of Chinese Medicine, Nanjing, China

${ }^{5}$ Department of Traumatology and Orthopedics, Affiliated Hospital of Nanjing University of Chinese Medicine, Nanjing, China

\#These authors contributed equally to this work.

\begin{abstract}
Introduction. The occurrence of osteoporosis (OP) has drawn considerable attention from scholars around the world due to the significant impacts thereof on the social economy and the quality of human life. OP research has been rapidly expanding since the inclusion of microRNAs (miRNAs) as critical regulators of gene-expression. However, despite the ability to evaluate miRNA gene therapy in OP being enhanced, there has been a scarcity of updated citation analyses that reflect such developments. In the present study, through bibliometric analysis, the global research activity and trends in regard to the relationship between OP and miRNAs were reviewed.

Methods. Publications related to miRNA and OP from 2000 to 2021 were retrieved via Web of Science Core Collection (WoSCC). The data included publication years, countries, journals, institutions, authors and keywords, and were sorted and summarized by bibliometrics, before being visually analyzed through VOS Viewer. Results. In the past five years, 599 articles have been published, with said studies accounting for $79.11 \%$ of all relevant documents, indicating the increased interest in the present research topic. The country with the highest contribution rate was China, and the publication rate of Journal of Bone and Mineral Research was the highest, followed by Bone. The institutions with the highest contribution rate were Nanjing Medical University. The most frequently occurring keywords were clustered into five groups. The research area of the first group described that circulating miRNA would be a potential biomarker for postmenopausal osteoporosis (PMOP). The remaining four groups involved the influences of miRNAs and exosomes on the osteogenic and adipogenic differentiation of mesenchymal stem cells (MSCs), and the interactions of lncRNA and miRNA with OP.
\end{abstract}

\footnotetext{
Correspondence address: Yang Guo and Yong Ma,

Laboratory of New Techniques of Restoration and

Reconstruction,

Institute of Traumatology and Orthopedics,

Nanjing University of Chinese Medicine,

Xianlin Street No. 138, Nanjing 210023, China

e-mails: drguoyang@njucm.edu.cn (YG);

mayong@njucm.edu.cn (YM)
}

This article is available in open access under Creative Common Attribution-Non-Commercial-No Derivatives 4.0 International (CC BY-NC-ND 4.0) license, allowing to download articles and share them with others as long as they credit the authors and the publisher, but without permission to change them in any way or use them commercially. 
Conclusions. The results of the present study will expand the research on miRNAs and OP. The research direction with the highest frequency was the miRNAs acting on osteoblasts and osteoclasts. The influence of miRNAs carried by exosomes on the differentiation of MSCs might become an effective method for OP cell-free treatment. (Folia Histochemica et Cytobiologica 2021, Vol. 59, No. 4, 203-211)

Key words: osteoporosis; miRNA; bibliometric analysis; visual analysis; exosomes

Abbreviations: OP — osteoporosis; miRNAs microRNAs; WoSCC - Web of Science Core Collection; PMOP — postmenopausal osteoporosis; MSCs - mesenchymal stem cells; AAY — average appearing year; IF — impact factor.

\section{Introduction}

Osteoporosis (OP) is a common bone metabolism disease. The imbalance between bone resorption and formation generates the piecemeal degradation of the bone microstructure and increases the risk of fragility fractures. Thus, OP is a significant threat to human health and the social economy. Complications such as severe fractures caused by OP are one of the primary accounts of morbidity and death in the elderly [1].

As endogenous small non-coding RNAs, microRNAs (miRNAs) regulate post-transcriptional silencing of target genes, and are about 18-25 nucleotides in length [2]. Numerous studies have found that up to $60 \%$ of human protein coding genes can be regulated by miRNAs [3], which bind to target genes in the 3'-untranslated regions (3'-UTR), and result in the degradation and transcription inhibition of mRNA [3, 4]. miRNAs mediate cell communication via exosomes, so as to regulate numerous biological processes including cell development, differentiation, proliferation, metabolism, and cell cycle regulation [3]. Additionally, miRNAs regulate osteoblast-dependent bone formation and osteoclast-related bone resorption [5, 6], and are potential therapeutic targets and biomarkers for OP.

Bibliometric analysis is a significant tool that can be used to find trends in various research fields and compare different countries, institutions, and research progress [7]. Despite the considerable significance of the relationship between miRNAs and OP, no qualitative and quantitative publication on such area has been demonstrated through bibliometric studies. Thus, in the present study, the aim was to use Web of Science to retrieve papers related to osteoporosis and miRNA via bibliometric analysis, categorize the countries, journals, authors, and institutions in which such studies are conducted, and regard frequently occurring keywords as the research object. Five research fields were selected and clarified, and analysis was conducted on the research progress and trends related to osteoporosis and miRNA.

\section{Search strategy}

Web of Science Core Collection (WoSCC) is widely used for analysis of the research trends and progress of medicine, and regarded as the best bibliometric database [8-11]. Thus, WoSCC was used in the present study. The following keywords and Boolean operators were used for the search: TS = ("Osteoporosis") AND $\mathrm{TS}=$ ("microRNA"” OR "miRNA"” OR "miR"”). Research published between 2000 and 2021 was selected, and included articles written in English. In order to avoid deviation due to the daily update of the database, the papers were retrieved on 15 February 2021.

The contents included title, author, country, publication journal, keywords and abstract, which were extracted and imported into Excel in .txt format. The Excel file was imported into VOSviewer 1.6.16 (Van Eck and Waltman, Leiden University, Leiden, The Netherlands) to analyze the relationship between the most cited study, and a cluster map was created for further visual analysis. Microsoft Excel 2019 (Microsoft Corporation, Redmond, WA, USA) was used for statistical analysis and creating graphs.

\section{Results}

In total, there were 1,180 studies published between 2000 and 2021. After screening, 827 papers were selected from the WoSCC, including 88 review articles $(10.64 \%)$. The total number of citations was 18,355 , and the average number of citations per item was 19.76. The search result flowchart is shown in Figure 1.

\section{Global publishing trends}

After Spencer et al. published the first study on OP and miRNA in the Journal of Bone and Mineral Research in 2008 [12], the number of related articles progressively grew from 2008 to 2012 and increased sharply after 2013 (Fig. 2). Significantly, 599 articles have been published in the past five years, with said studies accounting for $79.11 \%$ of all relevant documents, indicating the increased interest in the present research topic. 


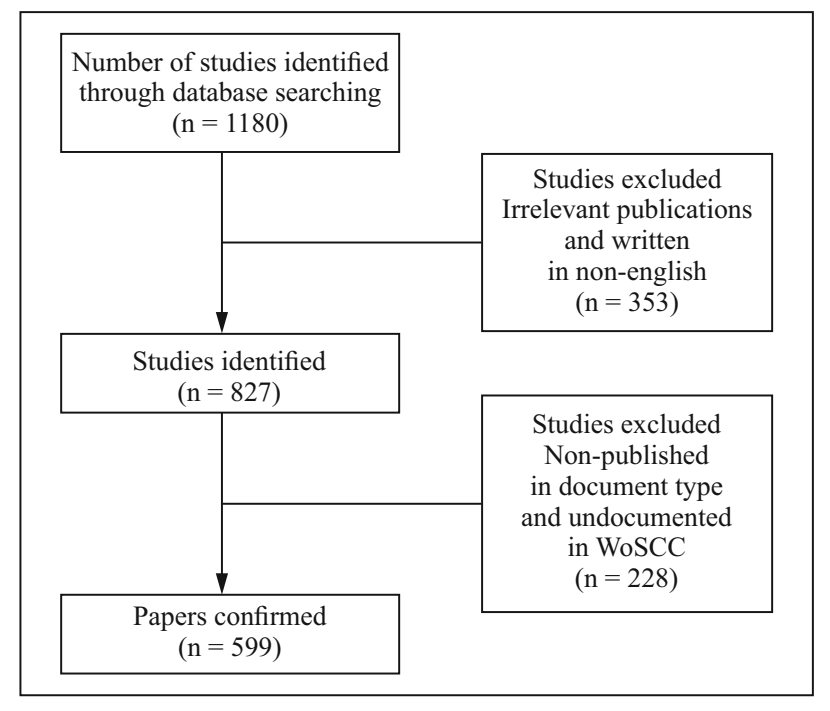

Figure 1. The flowchart of publications selection process used in this study. Document type include articles published in journal. Meeting abstract, editorial materials, book chapter, letter, proceedings paper, review, early access, correction, retracted publication was excluded.

\section{Bibliometric variables}

Bibliometric variables include relevant countries and institutions, the most cited authors, articles, and publications and information on the number of citations. VOSviewer was used to analyze the relevant literature.

The country with the highest number of publications was China with 395 published articles and 7710 citations. The institution with the highest contribution was Nanjing Medical University with 16 published and 552 citations. The most cited author was "Johannes Grillari", who worked at the University of Bodenkultur Wien, accounting for 26 publications in 14 journals. The most frequently cited research was "Differentially circulating miRNAs after recent osteoporotic fractures can influence osteogenic differentiation" [13] with 100 citations. Additional information is provided in Figures 3 and 4.

The journal with largest number of publications was Journal of Bone and Mineral Research (IF 6.741, 2020), with 49 articles and 1341 citations in total (Table 1). The most cited article was "MicroRNA-204 Regulates Runx2 Protein Expression and Mesenchymal Progenitor Cell Differentiation" [14] published by Huangjian et al. in Stem Cell (IF 6.277, 2020), with 412 citations.

The main research directions of miRNA and OP studies were based on cell biology, research experimental medicine, biochemistry molecular biology, endocrine metabolism, pharmacology and pharmacy. Among said research directions, studies involving cell biology had the greatest number of publications with a total of 169 (Additional file 1).

\section{Hotspots}

The keywords of 599 articles were analyzed in VOSviewer, and a keywords network was created to establish trends and current hotspots in the present research area. The keywords network reflected hotspots in the field of OP and miRNA [15]. Keywords that appeared more than 10 times were selected via title and abstract. Among 1866 keywords, 87 keywords were screened out and divided into five categories: red, yellow, green, blue and purple (Fig. 5a). The strongest keywords were classified into the red category, and included postmenopausal osteoporosis, bone density, circulating miRNA, and biomarkers; the green category included mesenchymal stem cells, osteogenic and adipogenic differentiation, and

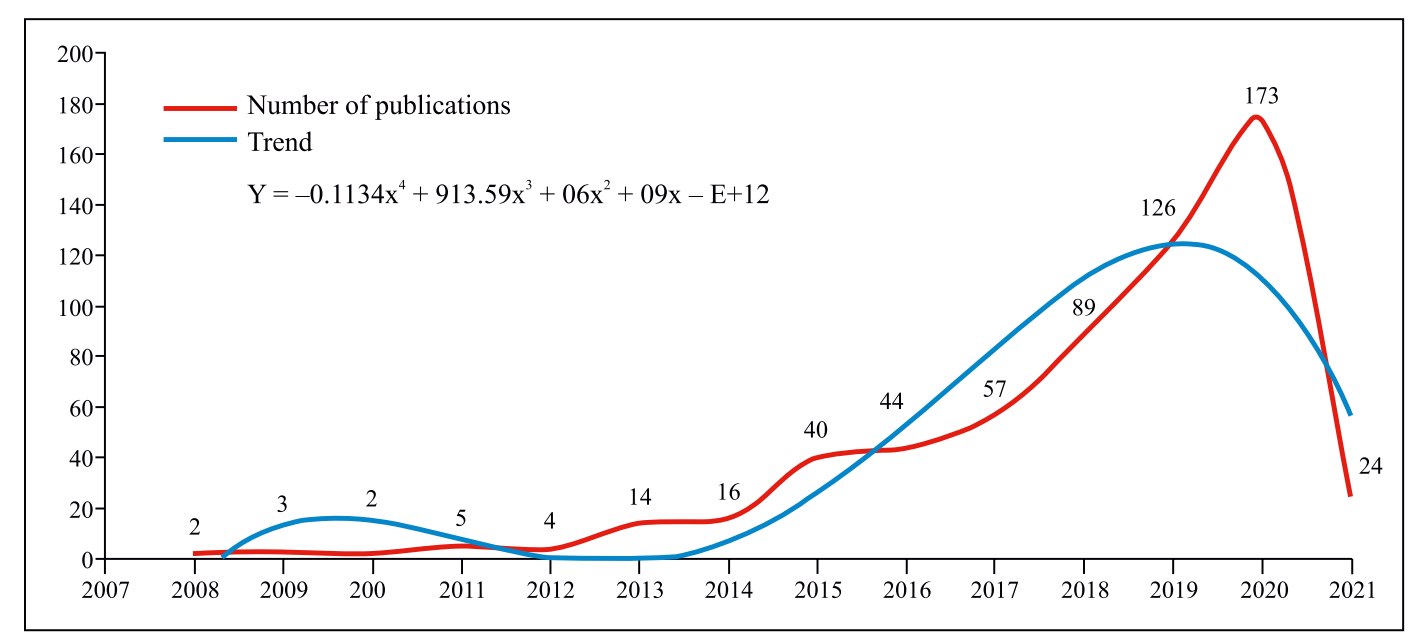

Figure 2. Numbers of publications about relation between OP and miRNA from 2008 to 2021. 
Table 1. Numbers of publications and Citation frequency for the relation between miRNA and OP of different sources

\begin{tabular}{|c|c|c|c|}
\hline Source & Number of publications & Frequency & IF 2020 \\
\hline Journal of Bone and Mineral Research (USA) & 49 & 1341 & 6.741 \\
\hline European Review for Medical and Pharmacological Sciences (Italy) & 35 & 254 & 3.507 \\
\hline Bone (USA) & 25 & 450 & 4.398 \\
\hline Molecular Medicine Reports (Greece) & 25 & 244 & 2.952 \\
\hline Experimental and Therapeutic Medicine (Greece) & 19 & 65 & 2.447 \\
\hline Journal of Cellular Biochemistry (USA) & 19 & 276 & 4.429 \\
\hline International Journal of Molecular Sciences (USA) & 16 & 153 & 5.923 \\
\hline Journal of Cellular Physiology (USA) & 16 & 223 & 6.384 \\
\hline Biochemical and Biophysical Research Communications (USA) & 14 & 127 & 3.575 \\
\hline International Journal of Molecular Medicine (USA) & 13 & 167 & 4.101 \\
\hline
\end{tabular}

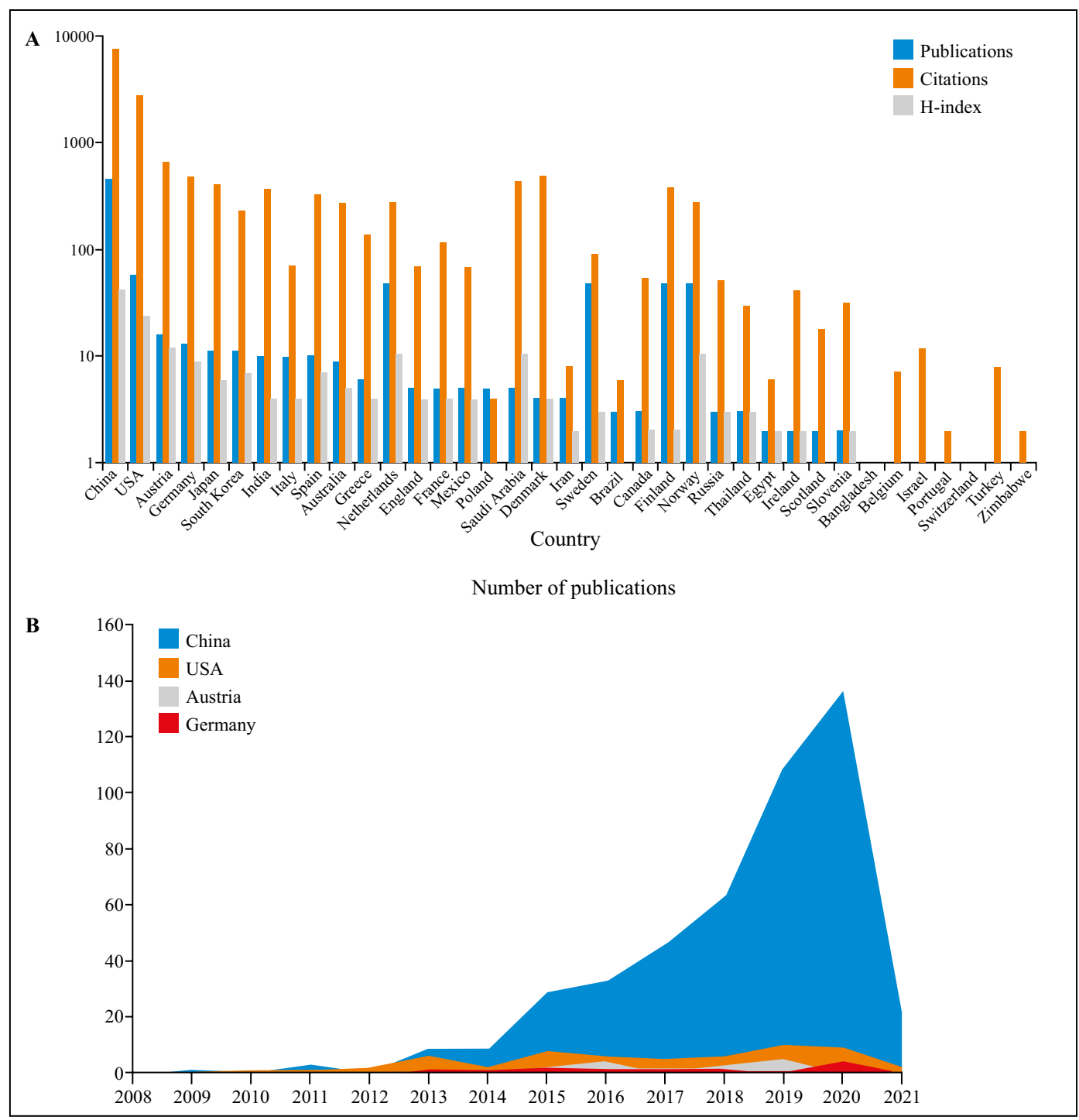

Figure 3. Numbers of publications and citation frequency for the miRNAs in OP-related of different countries. (a) The number of publications, citations and $\mathrm{H}$-index from different countries. (b) The number of publications from the top four countries per year. 


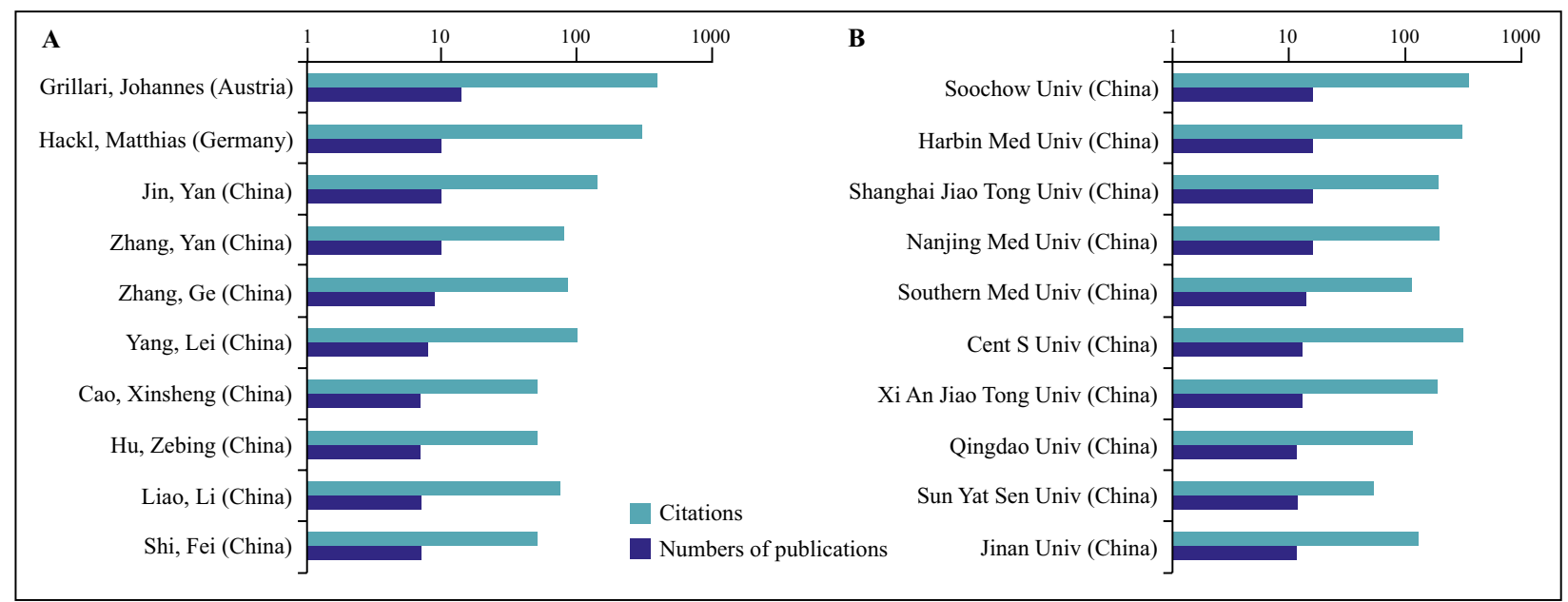

Figure 4. Distribution of authors and institutions for the miRNAs in OP-related. A. Numbers of publications and citation frequency for different authors. B. Numbers of publications and Citation frequency for different institutions.

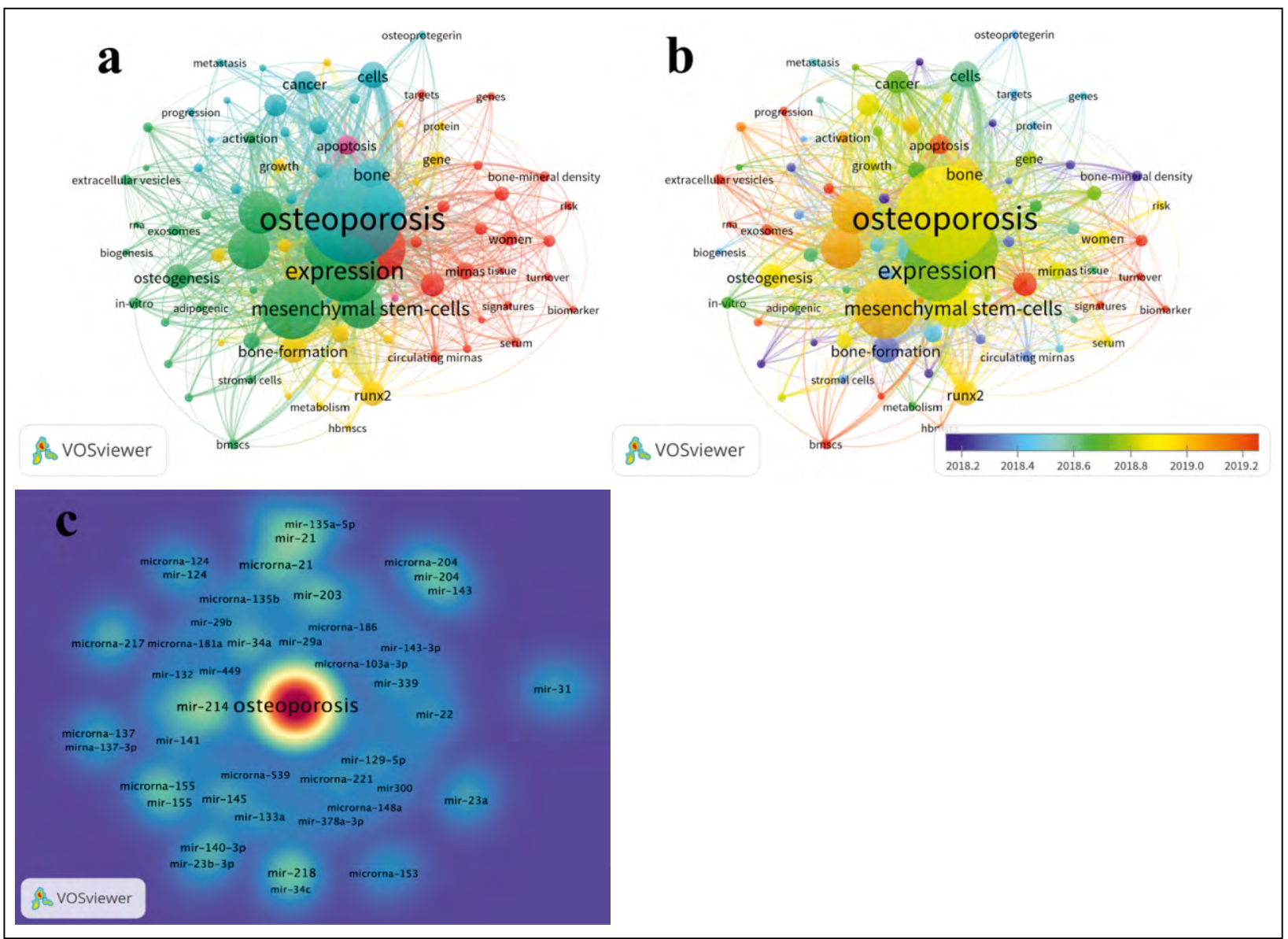

Figure 5. The keywords network related to miRNA and OP. A. The keywords were divided into five clusters and were portrayed by colors: cluster 1 ("osteoclast" and "rankl", in blue), cluster 2 ("circulating miRNA" and "biomarker", in red), cluster 3 ("mesenchymal stem-cell" and "adipogenic" and "osteogenesis" and "exosomes", in green), cluster 4 ("long non-cording RNA" and "bone-formation", in yellow), cluster 5 ("miR-214" and "apoptosis", in purple). In general, the larger size of a circle, the more frequently the keyword appears. The smaller distance between two terms, the larger number of co-occurrences appears. B. The earlier average appearing yearing of keyword appears in blue, the later in red. C. The larger number of adjacent items, the smaller distance between these and central keyword and the higher item density. The yellow or red direction indicated the higher density, the blue direction manifested the lower density. 
exosome; the yellow category included lncRNA and bone metabolism; the blue category included osteoclast and RANKL; and the purple category included miR-214 and apoptosis (Fig. 5a).

The different average appearing year (AAY) of keywords and developments of the theme over time in the map were analyzed and illustrated via VOSviewer. The average appearing years of keywords in the yellow and blue categories were more recent than that in the red category. In the red category, "long noncoding rna" and "exosomes" showed the relatively latest AAYs of 2019.45 and 2019.40, respectively (Additional file 2).

In addition, according to the frequency of occurrence in the article, the most cited miRNAs were selected and plotted as a density map (Fig. 5c). Among said miRNAs, miR-21, miR-155, miR-214, and miRNA-218 showed the highest project density (Additional file 3).

\section{Discussion}

As a huge economic burden, osteoporosis is a major public health problem. The annual number of osteoporosis-related fractures is expected to reach 4.83 million in China by 2035, which will cost approximately $\$ 19.92$ billion each year [16]. In the United States, more than 2 million osteoporosis-related fractures occur every year and the cost is estimated to exceed $\$ 25$ billion by 2025 [17]. Osteoporosis is preventive and remediable. Hence, finding more effective, safe and economical methods is of considerable research significance.

In the present study, a quantitative and qualitative analysis of global publications from 2008 to this year was firstly conducted, so as to determine the research progress and trends of miRNAs in osteoporosis-related research. For the aforementioned period, research on the relationship between OP and miRNA gradually increased. The country with the highest research contribution rate was China, the author with the highest research contribution rate was Johannes Grillari, and the journal with the highest research contribution rate was Journal of Bone and Mineral Research. Secondly, the most frequently occurring words were classified into five diverse categories, and the miRNAs in osteoporosis-related research activity and trends were systematically summarized. The latest potential research hotspots in the present research area appear to be "long noncoding RNA" and "exosomes".

In the past ten years, the number of publications has been increasing year by year and two trends have been exhibited: 2008 2013 was the initial stage, with a slow growth and fluctuation; and 2014 2021 was a period of rapid development, with future research expected to continue at a rapid pace.

Regarding countries, China and the USA were the regions that contributed the most, followed by Europe. In terms of the number of publications, the top ten authors and institutions were from China and Austria, but not the USA, which could be attributed to the scattered researchers and institutions in the USA. Among the top 10 authors, 2 were from the University of Bodenkultur Wien and TAmiRNA GmbH in Austria, respectively, and had a good cooperative relationship; 4 were from the Air Force Military Medical University in China; 2 were from Northwestern Polytechnical University and Hong Kong Baptist University in China, respectively. These authors and the institutions were the main research force in the present research field. Thus, establishing a good cooperative relationship between institutions might be a good choice.

For journals, a total of 946 journals published literature in the present research field. The top 10 journals with the most articles published $24.4 \%$ and the average IF was 4.446. Among said journals, three journals had an average of IF $>5$. Despite the large number of journals, no journal demonstrated a particular interest in the present research field, which made selecting journals difficult for the present authors. Among said journals, Journal of Bone and Mineral Research, Bone and Journal of Cellular Biochemistry could be regarded as core journals, and more research should be published therein.

The most cited article was "MicroRNA-204 Regulates Runx2 Protein Expression and Mesenchymal Progenitor Cell Differentiation" [14]. This study found that miR-204 could act on the 3'-untranslated region (3'-UTR) of Runx2 to down-regulate the expression of target proteins and genes then promote adipogenesis of mesenchymal progenitor cells and BMSCs. The second most co-cited reference was "A novel microRNA targeting HDAC5 regulates osteoblast differentiation in mice and contributes to primary osteoporosis in humans" [15]. This study revealed that the expression of miR-2861 decreased, while Runx2 levels decreased, inhibited osteoblast differentiation and promoted osteoporosis. The two aforementioned articles both explored the regulation of miRNA on Runx 2 to influence the differentiation of mesenchymal stem cells or osteoblasts. The two studies involved basic research in the present field, which every researcher should be familiar with.

With regard to scientific research trends, the application of miRNAs in osteoporosis has expanded from the initial osteogenic and adipogenesis of mesenchymal stem cells, osteoblasts, and osteoclasts 
to investigating the influence of circulatory miRNA, lncRNA and exosomes. For researchers, with the continuous development of research, the molecular mechanism of osteogenesis and adipogenesis of MSCs will be more distinct. The positive and negative feedback and bidirectional regulation of miRNAs on transcription factors and proteins has been demonstrated to be related to osteogenic and adipogenic differentiation of MSCs [18-23], and the effect of miRNA gene therapy targets for OP has significant potential. Most studies have demonstrated the ability of miRNAs to promote osteogenic differentiation of MSCs, and the significant effects thereof. However, due to the lack of ability to target tissues and the instability of being degraded by nucleases, only a few preclinical studies have shown that miRNAs could be directly injected and delivered into bone tissue. Hence, finding more miRNAs with positive effects on bone metabolism and constructing a more stable, safe and effective delivery systems are urgent problems that must be solved to improve OP treatment via targeting miRNAs gene therapy. An area worthy of further study is determining whether the insufficiency of undemanding degradation and poor targeting ability can be remedied through structural modification, capsule shells or scaffolds. Secondly, exosomal miRNAs from different sources have been shown to target different genes and proteins to regulate osteogenic differentiation [25, 26]. Compared with stem cell therapy, exosomes have higher safety and the ability to penetrate the blood-brain barrier to treat brain injuries [26, 27], thereby exhibiting a wider range of disease treatment effects. Exosomes can also be loaded with miRNAs for targeted treatment of OP, showing significant research potential. Although several clinical trials have been conducted in existing research, due to the lack of basic knowledge of exosomes research methods, there is currently no clinically approved exosomes treatment method. Whether exosomal miRNAs can make up for the deficiencies of miRNAs, which are easily degraded by nucleases in delivery systems, and whether exosomes can be used to treat orthopedic diseases need further research to determine.

Further, studies on preforming serum circulating miRNAs as OP biomarkers are increasing. The stability, measurability and effects of circulation miRNAs on bone metabolism promote new directions in the diagnosis and prognosis of OP [28-31]. However, individual differences such as gender, age, lifestyle, and disease need to be considered. The standard and quality of detection are still unspecified, and determining how to implement regulations, and promote the measuring mode and quality control needs further research.
At present, several effective drugs such as estrogen, denosumab, and bisphosphonates have been approved to treat OP. The function of these drugs is to inhibit the formation of osteoclasts. Similarly, numerous studies have revealed that miRNAs acted on osteoclasts to regulate bone metabolism and had an anti-osteoporotic effect $[32,33]$. Additionally, lncRNA [34-36] and several natural medicine active ingredients [37] could target miRNAs to promote the apoptosis of osteoclasts. In recent years, with increasing attention being paid to the quality of life and health, natural medicines have become the focus of anti-osteoporosis drug research due to the safety and lower cost thereof. Whether to explore safer and more effective active ingredients of natural medicines that target miRNAs has also become another new direction for OP treatment worthy of further study.

Regarding the miRNAs density map, miR-21 [38], miR155 [39, 40], miR-204 [41] and miRNA-218 [42] exhibited the highest research frequency, and could be used as potential target genes for the biomarkers or treatment of OP.

\section{Limitations}

There were several limitations in the present study. First, non-recorded publications in the WoSCC were not extracted. Databases such as Scopus, Embase, PubMed, and others were not used for analysis. Secondly, the selected studies were written in English, and relevant information in other languages was not included. Further, self-reference bias was not considered in the present study. Third, deviations due to the daily update of the database were likely not avoided, since new research will be regularly added.

Despite the limitations, the present bibliometric analysis adds to the present understanding of the frontiers and development trends of miRNAs in osteoporosis-related research.

\section{Conclusions}

miRNAs are the newest effective tool to act as biomarkers and pharmacological targets in OP, and have promising application prospects in treatment. Over the past decade, research has continued to grow. Asian countries exhibited higher contribution rates. Scholars are widely distributed around the world, and more cooperation between top authors and institutions is needed. In addition, the notable research hotspots were "exosomes", "circulating miRNA" and "IncRNA".

\section{Funding}

This study was supported by National Natural Science Foundation of China (82074458); "Qing Lan Project" 
of Jiangsu University Funding Project (Su Teacher [2018] No.12); Graduate Research and Innovation Program of Jiangsu Province (KYCX20_1461); Project Funded by the Priority Academic Program Development of Jiangsu Higher Education Institutions (Integration of Chinese and Western Medicine) ([2018] No.87).

\section{Ethical approval and consent to participate}

Not applicable.

\section{Consent for publication}

Not applicable.

\section{Availability of data and materials}

All data generated or analyzed during this study are included in this published article.

\section{Authors' contributions}

Conceived and designed the experiments: Yang Guo and Yong Ma; Performed the experiments: Li Yun and Lining Wang; Identified studies for inclusion and data extraction: Li Yun, Lining Wang, Qiuge Han and Jie Sun; Data analyses and assessing study quality: Li Yun, Yalan Pan and MengMin Liu; Performing data analyses and first draft of manuscript: Li Yun, Lining Wang; Advising and critically revising the manuscript: Guanglu Yang and Jirimutu Xiao. All authors agree to be accountable for the work and to ensure that any questions relating to the accuracy and integrity of the paper are investigated and properly resolved.

\section{Conflict of interests}

The authors declare that they have no competing interests.

\section{References}

1. Rachner TD, Khosla S, Hofbauer LC. Osteoporosis: now and the future. Lancet. 2011; 377(9773): 1276-1287, doi: 10.1016/ S0140-6736(10)62349-5, indexed in Pubmed: 21450337.

2. Ge DW, Wang WW, Chen HT, et al. Functions of microRNAs in osteoporosis. Eur Rev Med Pharmacol Sci. 2017; 21(21): 4784-4789, indexed in Pubmed: 29164586.

3. Feng Q, Zheng S, Zheng J. The emerging role of microRNAs in bone remodeling and its therapeutic implications for osteoporosis. Biosci Rep. 2018; 38(3), doi: 10.1042/BSR20180453, indexed in Pubmed: 29848766.

4. Yoon JH, Kim J, Gorospe M. Long noncoding RNA turnover. Biochimie. 2015; 117: 15-21, doi: 10.1016/j.biochi.2015.03.001, indexed in Pubmed: 25769416.

5. van Wijnen AJ, van de Peppel J, van Leeuwen JP, et al. MicroRNA functions in osteogenesis and dysfunctions in os- teoporosis. Curr Osteoporos Rep. 2013; 11(2): 72-82, doi: 10.1007/s11914-013-0143-6, indexed in Pubmed: 23605904.

6. Bellavia D, De Luca A, Carina V, et al. Deregulated miRNAs in bone health: Epigenetic roles in osteoporosis. Bone. 2019; 122: 52-75, doi: 10.1016/j.bone.2019.02.013, indexed in Pubmed: 30772601.

7. Khan A, Choudhury N, Uddin S, et al. Longitudinal trends in global obesity research and collaboration: a review using bibliometric metadata. Obes Rev. 2016; 17(4): 377-385, doi: 10.1111/obr.12372, indexed in Pubmed: 26864566.

8. Zhao N, Tao K, Wang G, et al. Global obesity research trends during 1999 to 2017: A bibliometric analysis. Medicine (Baltimore). 2019; 98(4): e14132, doi: 10.1097/ MD.0000000000014132, indexed in Pubmed: 30681576.

9. Devos P, Menard J. Bibliometric analysis of research relating to hypertension reported over the period 1997 2016. J Hypertens. 2019; 37(11): 2116-2122, doi: 10.1097/ HJH.0000000000002143, indexed in Pubmed: 31136459.

10. Berlinberg A, Bilal J, Riaz IB, et al. The 100 top-cited publications in psoriatic arthritis: a bibliometric analysis. Int J Dermatol. 2019; 58(9): 1023-1034, doi: 10.1111/ijd.14261, indexed in Pubmed: 30315571.

11. Guo S, Wang Li, Xie Y, et al. Bibliometric and Visualized Analysis of Stem Cells Therapy for Spinal Cord Injury Based on Web of Science and CiteSpace in the Last 20 Years. World Neurosurg. 2019; 132: e246-e258, doi: 10.1016/j. wneu.2019.08.191, indexed in Pubmed: 31493613.

12. Luzi E, Marini F, Sala SC, et al. Osteogenic differentiation of human adipose tissue-derived stem cells is modulated by the miR-26a targeting of the SMAD1 transcription factor. J Bone Miner Res. 2008; 23(2): 287-295, doi: 10.1359/jbmr.071011, indexed in Pubmed: 18197755.

13. Weilner S, Skalicky S, Salzer B, et al. Differentially circulating miRNAs after recent osteoporotic fractures can influence osteogenic differentiation. Bone. 2015; 79: 43-51, doi: 10.1016/j. bone.2015.05.027, indexed in Pubmed: 26026730.

14. Huang J, Zhao L, Xing L, et al. MicroRNA-204 regulates Runx 2 protein expression and mesenchymal progenitor cell differentiation. Stem Cells. 2010; 28(2): 357-364, doi: 10.1002/ stem.288, indexed in Pubmed: 20039258.

15. Li H, Xie H, Liu W, et al. A novel microRNA targeting HDAC5 regulates osteoblast differentiation in mice and contributes to primary osteoporosis in humans. J Clin Invest. 2009; 119(12): 3666-3677, doi: 10.1172/JCI39832, indexed in Pubmed: 19920351.

16. Zeng Q, Li Na, Wang Q, et al. The Prevalence of Osteoporosis in China, a Nationwide, Multicenter DXA Survey. J Bone Miner Res. 2019; 34(10): 1789-1797, doi: 10.1002/jbmr.3757, indexed in Pubmed: 31067339.

17. Burge R, Dawson-Hughes B, Solomon DH, et al. Incidence and economic burden of osteoporosis-related fractures in the United States, 2005-2025. J Bone Miner Res. 2007; 22(3): 465-475, doi: 10.1359/jbmr.061113, indexed in Pubmed: 17144789.

18. Xiao J, Qin S, Li W, et al. Osteogenic differentiation of rat bone mesenchymal stem cells modulated by MiR186 via SIRT6. Life Sci. 2020; 253: 117660, doi: 10.1016/j. lfs.2020.117660, indexed in Pubmed: 32294474.

19. Xiao Ye, Guo Qi, Jiang TJ, et al. miR-483-3p regulates osteogenic differentiation of bone marrow mesenchymal stem cells by targeting STAT1. Mol Med Rep. 2019; 20(5): 4558-4566, doi: 10.3892/mmr.2019.10700, indexed in Pubmed: 31702021.

20. Tan K, Peng YT, Guo P. MiR-29a promotes osteogenic differentiation of mesenchymal stem cells via targeting HDAC4. Eur Rev Med Pharmacol Sci. 2018; 22(11): 3318-3326, 
doi: 10.26355/eurrev_201806_15151, indexed in Pubmed: 29917181.

21. Zha JP, Wang XQ, Di J. MiR-920 promotes osteogenic differentiation of human bone mesenchymal stem cells by targeting HOXA7. J Orthop Surg Res. 2020; 15(1): 254, doi: 10.1186/ s13018-020-01775-7, indexed in Pubmed: 32650806.

22. Lin $\mathrm{Z}, \mathrm{He} \mathrm{H}$, Wang $\mathrm{M}$, et al. MicroRNA-130a controls bone marrow mesenchymal stem cell differentiation towards the osteoblastic and adipogenic fate. Cell Prolif. 2019; 52(6): e12688, doi: 10.1111/cpr.12688, indexed in Pubmed: 31557368.

23. Kong J, Wan LP, Liu ZM, et al. MiR-1301 promotes adipogenic and osteogenic differentiation of BMSCs by targeting Satb2. Eur Rev Med Pharmacol Sci. 2020; 24(7): 3501-3508, doi: 10.26355/eurrev_202004_20809, indexed in Pubmed: 32329823

24. Yang JX, Xie P, Li YS, et al. Osteoclast-derived miR-23a$5 p$-containing exosomes inhibit osteogenic differentiation by regulating Runx2. Cell Signal. 2020; 70: 109504, doi: 10.1016/j. cellsig.2019.109504, indexed in Pubmed: 31857240.

25. Yu L, Sui B, Fan W, et al. Exosomes derived from osteogenic tumor activate osteoclast differentiation and concurrently inhibit osteogenesis by transferring COL1A1-targeting miRNA-92a-1-5p. J Extracell Vesicles. 2021; 10(3): e12056, doi: 10.1002/jev2.12056, indexed in Pubmed: 33489015.

26. Andaloussi SEL, Lakhal S, Mäger I, et al. Exosomes for targeted siRNA delivery across biological barriers. Advanced Drug Delivery Reviews. 2013; 65(3): 391-397, doi: 10.1016/j. addr.2012.08.008, indexed in Pubmed: 22921840.

27. Janas T, Janas MM, Sapoń K, et al. Mechanisms of RNA loading into exosomes. FEBS Lett. 2015; 589(13): 1391-1398, doi: 10.1016/j.febslet.2015.04.036, indexed in Pubmed: 25937124.

28. Ma J, Lin X, Chen C, et al. Circulating miR-181c-5p and miR497-5p Are Potential Biomarkers for Prognosis and Diagnosis of Osteoporosis. J Clin Endocrinol Metab. 2020; 105(5), doi: 10.1210/clinem/dgz300, indexed in Pubmed: 31872255 .

29. Ismail SM, El Boghdady NA, Hamoud HS, et al. Evaluation of circulating miRNA-208a-3p, miRNA-155-5p and miRNA-637 as potential non-invasive biomarkers and the possible mechanistic insights into pre- and postmenopausal osteoporotic females. Arch Biochem Biophys. 2020; 684: 108331, doi: 10.1016/j.abb.2020.108331, indexed in Pubmed: 32151564.

30. Ding W, Ding S, Li J, et al. Aberrant Expression of miR-100 in Plasma of Patients with Osteoporosis and its Potential Diagnostic Value. Clin Lab. 2019; 65(9), doi: 10.7754/Clin. Lab.2019.190327, indexed in Pubmed: 31532098.

31. Ramírez-Salazar EG, Carrillo-Patiño S, Hidalgo-Bravo A, et al. Serum miRNAs miR-140-3p and miR-23b-3p as potential biomarkers for osteoporosis and osteoporotic fracture in postmenopausal Mexican-Mestizo women. Gene. 2018; 679: 19-27, doi: 10.1016/j.gene.2018.08.074, indexed in Pubmed: 30171938 .
32. Cong C, Tian J, Gao T, et al. IncRNA GAS5 Is Upregulated in Osteoporosis and Downregulates miR-21 to Promote Apoptosis of Osteoclasts. Clin Interv Aging. 2020; 15: 1163-1169, doi: 10.2147/CIA.S235197, indexed in Pubmed: 32764903.

33. Madhyastha R, Madhyastha $\mathrm{H}$, Pengjam $\mathrm{Y}$, et al. The pivotal role of microRNA-21 in osteoclastogenesis inhibition by anthracycline glycoside aloin. J Nat Med. 2019; 73(1): 59-66, doi: 10.1007/s11418-018-1237-3, indexed in Pubmed: 30116953.

34. Feng J, Wang JX, Li CH. LncRNA GAS5 overexpression alleviates the development of osteoporosis through promoting osteogenic differentiation of MSCs via targeting microRNA-498 to regulate RUNX2. Eur Rev Med Pharmacol Sci. 2019; 23(18): 7757-7765, doi: 10.26355/eurrev_201909_18985, indexed in Pubmed: 31599401.

35. Zhang $\mathrm{N}, \mathrm{Hu} \mathrm{X}, \mathrm{He} \mathrm{S}$, et al. LncRNA MSC-AS1 promotes osteogenic differentiation and alleviates osteoporosis through sponging microRNA-140-5p to upregulate BMP2. Biochem Biophys Res Commun. 2019; 519(4): 790-796, doi: 10.1016/j. bbrc.2019.09.058, indexed in Pubmed: 31551149.

36. Yang X, Yang J, Lei P, et al. LncRNA MALAT1 shuttled by bone marrow-derived mesenchymal stem cells-secreted exosomes alleviates osteoporosis through mediating microRNA-34c/SATB2 axis. Aging (Albany NY). 2019; 11(20): 8777-8791, doi: 10.18632/aging.102264, indexed in Pubmed: 31659145

37. Madhyastha R, Madhyastha H, Pengjam Y, et al. The pivotal role of microRNA-21 in osteoclastogenesis inhibition by anthracycline glycoside aloin. J Nat Med. 2019; 73(1): 59-66, doi: 10.1007/s11418-018-1237-3, indexed in Pubmed: 30116953.

38. Jiang LB, Tian L, Zhang CG. Bone marrow stem cells-derived exosomes extracted from osteoporosis patients inhibit osteogenesis via microRNA-21/SMAD7. Eur Rev Med Pharmacol Sci. 2018; 22(19): 6221-6229, doi: 10.26355/eurrev 201810 16028, indexed in Pubmed: 30338786.

39. Zhang J, Zhao H, Chen J, et al. Interferon- $\beta$-induced miR155 inhibits osteoclast differentiation by targeting SOCS1 and MITF. FEBS Lett. 2012; 586(19): 3255-3262, doi: 10.1016/j. febslet.2012.06.047, indexed in Pubmed: 22771905.

40. Ren C, Li M, Sun L, et al. Serum MicroRNA Differences Between Fracture in Postmenopausal Women with and without Diabetes. Orthop Surg. 2021; 13(1): 285-295, doi: 10.1111/ os.12866, indexed in Pubmed: 33283469.

41. Sacchetti B, Fatica A, Sorci M, et al. Effect of miR-204\&211 and RUNX2 control on the fate of human mesenchymal stromal cells. Regen Med Res. 2017; 5: 2, doi: 10.1051/rmr/170004, indexed in Pubmed: 29206625.

42. Li X, Gibson G, Kim JS, et al. MicroRNA-146a is linked to pain-related pathophysiology of osteoarthritis. Gene. 2011; 480(1-2): 34-41, doi: 10.1016/j.gene.2011.03.003, indexed in Pubmed: 21397669.

Submitted: 23 June, 2021

Accepted after reviews: 8 November, 2021 Available as AoP: 1 December, 2021 